\title{
Prasugrel overcomes high on-clopidogrel platelet reactivity in the acute phase of acute coronary syndrome and maintains its antiplatelet potency at 30-day follow-up
}

\author{
Marek Koziński ${ }^{1}$, Karolina Obońska ${ }^{1,2}$, Katarzyna Stankowska ${ }^{3}$, Eliano Pio Navarese ${ }^{1}$, \\ Tomasz Fabiszak ${ }^{1}$, Wioleta Stolarek ${ }^{1,2}$, Michał Kasprzak ${ }^{1,2}$, Jolanta Maria Siller-Matula ${ }^{4}$ \\ Danuta Rość ${ }^{3}$, Jacek Kubica ${ }^{1}$, Stefano De Servi ${ }^{5}$ \\ ${ }^{1}$ Department of Cardiology and Internal Medicine, Collegium Medicum, \\ Nicolaus Copernicus University, Bydgoszcz, Poland \\ ${ }^{2}$ Department of Pharmacology and Therapeutics, Collegium Medicum, \\ Nicolaus Copernicus University, Bydgoszcz, Poland \\ ${ }^{3}$ Department of Pathophysiology, Collegium Medicum, Nicolaus Copernicus University, Bydgoszcz, Poland \\ ${ }^{4}$ Department of Cardiology, Medical University of Vienna, Vienna, Austria \\ ${ }^{5}$ Coronary Care Unit, IRCCS Policlinico S. Matteo, Pavia, Italy
}

\begin{abstract}
Background: The aim of this study was to assess antiplatelet effect of prasugrel in acute coronary syndrome (ACS) patients with high on-treatment platelet reactivity (HTPR) on clopidogrel, undergoing percutaneous coronary intervention (PCI).

Methods: A prospective, platelet reactivity-guided, parallel-group, open-label study including 71 patients pretreated with clopidogrel $600 \mathrm{mg}$ and assigned either to prasugrel (30 mg loading dose, 10 mg maintenance dose; $n=46)$ or clopidogrel $(150 \mathrm{mg}$ maintenance dose for 6 days and thereafter $75 \mathrm{mg}$ maintenance dose; $n=25)$ regimen, based on vasodilator-stimulated phosphoprotein (VASP)-assessed platelet reactivity index (PRI; > 50\% vs. $\leq 50 \%$ ) measured next morning post-PCI.
\end{abstract}

Results: Median PRI value after switch to prasugrel sharply declined at 24 h (70.0 [61.3$-75.6] v s .11 .9[6.8-25.7] \% ; p<0.000001)$ and slightly but significantly rose between $24 \mathrm{~h}$ and 30 days (27.9 [15.5-46.8]\%; $p<0.0006)$. In contrast, median PRI values in the clopidogrel group were similar at baseline and at $24 \mathrm{~h}$ (25.1 [13.7-40.2] vs. 22.0 [18.4-36.8]\%; $p=N S)$ and then modestly rose at 30 days (30.3 [20.4-45.7]\%; $p<0.03)$. The prevalence of HTPR decreased in the prasugrel group between baseline and $24 \mathrm{~h}$ measurements (100.0 vs. 4.3\%; $p<0.0001$ ). Rates of patients with HTPR at $24 \mathrm{~h}$ and 30 days were similar in both groups, so were the tendencies in patterns of platelet inhibition evaluated with multiple electrode aggregometry as compared with the VASP assay.

Conclusions: Our study indicates that prasugrel overcomes HTPR on clopidogrel in the acute phase of interventionally treated ACS and maintains its antiplatelet potency in 30-day follow-up. Potential clinical benefits of personalized antiplatelet prasugrel-based therapy warrant further investigation in clinical ACS trials. (Cardiol J 2014; 21, 5: 547-556)

Key words: prasugrel, clopidogrel, high on-treatment platelet reactivity, VASP assay, multiple electrode aggregometry

Address for correspondence: Marek Koziński, MD, PhD, Department of Cardiology and Internal Medicine, Collegium Medicum, Nicolaus Copernicus University, ul. Sklodowskiej-Curie 9, 85-094 Bydgoszcz, Poland, tel: +48 52 5854023, fax: +4852 5854024, e-mail: marekkozinski@wp.pl

Received: 10.11.2013 Accepted: 17.03.2014 


\section{Introduction}

The modest potency and large interindividual variability of clopidogrel may result in its insufficient antiplatelet effect in some patient subsets $[1,2]$. Subsequently, subjects with high on-treatment platelet reactivity (HTPR) on clopidogrel are exposed to increased risk of cardiovascular (CV) events in the setting of percutaneous coronary intervention (PCI) [3].

Prasugrel is associated with a faster onset of action and enhanced inhibition of platelet aggregation than clopidogrel and was shown to reduce CV events more effectively than clopidogrel in patients with acute coronary syndrome (ACS) undergoing PCI $[4,5]$.

Assuming that a $30 \mathrm{mg}$ loading dose of prasugrel followed by a $10 \mathrm{mg}$ maintenance dose would produce an efficient platelet $\mathrm{P} 2 \mathrm{Y}_{12}$ receptor blockade in ACS patients treated with PCI and resistant to the initial $600 \mathrm{mg}$ loading dose of clopidogrel, we aimed in the present study to test the antiplatelet effect of prasugrel in interventionally treated ACS patients with HTPR.

\section{Methods}

\section{Study population and design}

The study was designed as a prospective, single-center, non-randomized, investigator-initiated, platelet reactivity-guided, parallel-group, openlabel trial. Consecutive patients admitted to The Department of Cardiology and Internal Medicine between 15 November 2010 and 31 December 2011 were screened with respect to their eligibility for the study enrolment. Inclusion criteria were as follows: age between 18 and 74 years, ACS successfully treated with PCI with stent implantation, pre-treatment with loading doses of clopidogrel (600 $\mathrm{mg}$ ) and aspirin (300 $\mathrm{mg})$, and platelet function assessment available between 12 and $24 \mathrm{~h}$ after PCI. Patients were excluded from the study in the presence of at least of one of the following criteria: age equal or above 75 years, prior transient ischemic attack or stroke, body weight less than $60 \mathrm{~kg}$, contraindication for aspirin and/or thienopyridines, the need for anticoagulation in the following month or prior chronic anticoagulation, chronic treatment with a thienopyridine or non-thienopyridine platelet $\mathrm{P} 2 \mathrm{Y}_{12}$ receptor blocker, necessity for coronary artery bypass grafting in the following month, current bleeding disorder, active bleeding event, active peptic ulcer disease, history of gastrointestinal bleeding within the prior month, severe liver failure (Child Pugh class C), hemoglobin level at presentation below $90 \mathrm{~g} / \mathrm{L}$, platelet count below 100 000/mL and ongoing pregnancy.

Based on the initial platelet function assessment performed on the next morning following PCI, patients were assigned to one of 2 particular thienopyridine regimens (Fig. 1). Blood samples were collected at 10.00 a.m. as we previously demonstrated that platelet inhibition by clopidogrel is weaker in the midmorning hours [6]. Subjects with HTPR on clopidogrel defined as the platelet reactivity index (PRI) above $50 \%$ were immediately loaded with $30 \mathrm{mg}$ of prasugrel with a subsequent maintenance dose of $10 \mathrm{mg}$ q.d. until the end of the study. Patients with adequate platelet inhibition $(\mathrm{PRI} \leq 50 \%)$ received a $150 \mathrm{mg}$ maintenance dose of clopidogrel q.d. for 6 days and thereafter a $75 \mathrm{mg}$ maintenance dose q.d.

All participants provided informed written consents for participation in the study. The study protocol was approved by the Local Ethics Committee in accordance with the Declaration of Helsinki.

\section{Coronary arteriography and PCI}

Interventional procedures were performed according to current guidelines [7]. Abciximab or eptifibatide were administered at the discretion of the invasive cardiologist in doses recommended by the drug manufacturers.

\section{Assessment of platelet reactivity}

We applied 2 independent methods to assess platelet reactivity.

A standardized flow cytometric assay (VASP; BioCytex, Marseille, France) was used to determine the inhibition by adenosine diphosphate (ADP) of phosphorylation of vasodilator-stimulated phosphoprotein (VASP), which is mediated by the $\mathrm{P}_{2} \mathrm{Y}_{12}$ receptor through the inhibition of adenylyl cyclase. The VASP assay is regarded as the most specific tool assessing the effect of thienopyridine or non-thienopyridine blockers on the platelet $\mathrm{P}_{2} \mathrm{Y}_{12}$ receptor. Platelet reactivity was expressed as PRI calculated as a percentage according to the formula: $\left[\left(\mathrm{GMFI}_{(\mathrm{PGEl})}-\mathrm{GMFI}_{(\mathrm{PGE} 1+\mathrm{ADP})}\right) / \mathrm{GMFI}_{(\mathrm{PGEI})}\right]$ $\times 100$. The ratio is expressed as mean percentage platelet reactivity, inversely correlated with clopidogrel or prasugrel treatment efficiency. The normal value of PRI without treatment with ADP antagonists is $69-100 \%$ [8].

Whole blood aggregation was determined using multiple electrode aggregometry (MEA) on a new generation impedance aggregometer (Multiplate Analyzer, Verum Diagnostica GmbH, Munich, Germany) according to the manufacturer's instructions. The instrument detects the impe- 


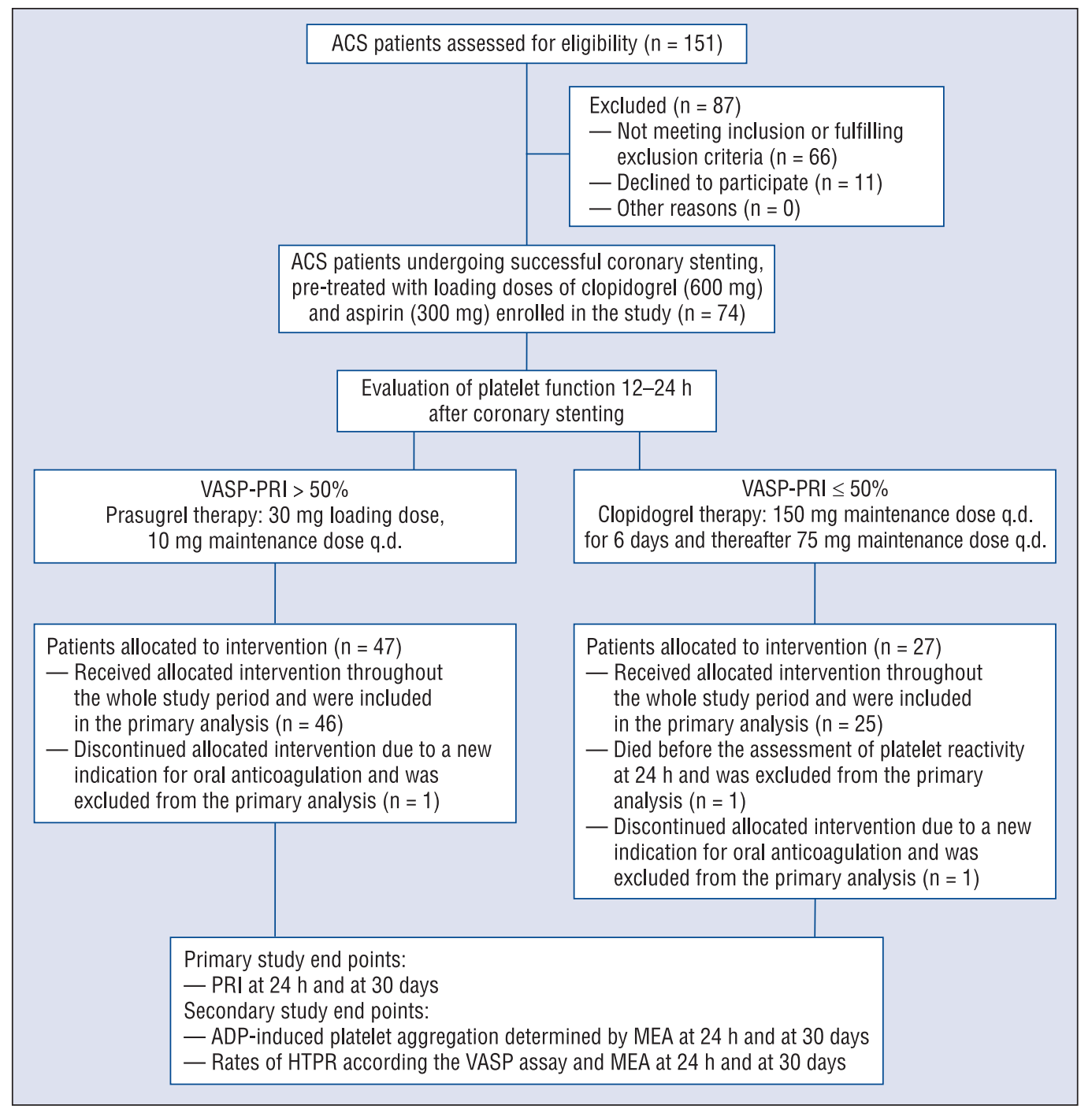

Figure 1. Flow diagram of the study; ACS — acute coronary syndrome; ADP — adenosine diphosphate; HTPR — high on-clopidogrel platelet reactivity; MEA — multiple electrode aggregometry; PRI — platelet reactivity index; VASP — vasodilator-stimulated phosphoprotein.

dance change related to platelet aggregation and transforms it into arbitrary aggregation units (AU) that are plotted against time. The area under the aggregation curve (AUC) is an estimator of platelet aggregation that was evaluated in our study. Aggregation, quantified as the AUC, is displayed in arbitrary units $(10 \mathrm{AU} \times \min =1 \mathrm{U})$ [9]. To assess the platelet response to clopidogrel or prasugrel, we applied ADP-induced aggregation. A good reproducibility of MEA, with the variability of results $<6 \%$, was reported by Tóth et al. [9].

\section{Study definitions and endpoints}

The primary HTPR definitions (PRI $>50 \%$ and AUC $>46.8 \mathrm{U}$ for VASP and MEA, respecti- vely) were based on the consensus document of the Working Group on On-Treatment Platelet Reactivity and prior studies indicating a strong link between increased platelet reactivity and the occurrence of stent thrombosis and recurrent ischemic events [1, 10-15]. To strengthen our findings we also applied in the calculations other clinically validated HTPR definitions (PRI $>60 \%[16,17]$ and AUC $>54$ U [18] for VASP and MEA, respectively).

Values of PRI measured $24 \mathrm{~h}$ and 30 days after assignment to the particular thienopyridine regimen constituted the primary study end points, while values of ADP-induced platelet aggregation determined by MEA, rates of HTPR according the VASP assay and MEA, all at $24 \mathrm{~h}$ and at 30 days 
were selected as the secondary study end points. MEA measurements at baseline and after $24 \mathrm{~h}$ were restricted only to the patients not receiving glycoprotein (GP) IIb/IIIa inhibitors. Blood samples for platelet function assessment at $24 \mathrm{~h}$ and at 1 month were collected at 10.00 a.m. Additionally, we prespecified to report all cases of death, new onset myocardial infarction, definite stent thrombosis, new onset chronic heart failure and bleeding events of any severity occurring in the study participants during the study period.

\section{Statistical analysis and sample size calculation}

Based on a study conducted by Bonello et al. [14] we assumed the mean value of PRI in HTPR patients pretreated with clopidogrel $600 \mathrm{mg}$ of $68.5 \pm 10.3 \%$. Similarly, we expected that additional administration of $30 \mathrm{mg}$ of prasugrel in HTPR patients who had received a $600 \mathrm{mg}$ loading dose of clopidogrel would decrease PRI to a value comparable with that observed in clopidogrel-naive patients after treatment with a $60 \mathrm{mg}$ loading dose of prasugrel (34.3 $\pm 23.1 \%$ ) [19]. Additionally, we anticipated in our prasugrel-treated patients to obtain PRI values at 30 days comparable to these observed in the TRITON-TIMI 38 platelet substudy at this time point $(33.6 \pm 2.9 \%)$ [20]. Bearing in mind these results and assuming a 2 -sided alpha value of 0.05 , we calculated, using the t-test for dependent variables, that enrolment of 46 HTPR patients would provide a $99.9 \%$ power to demonstrate significant reductions in PRI associated with the implementation of prasugrel-based antiplatelet regimen between its baseline value and both values at $24 \mathrm{~h}$ and 30 days.

The Shapiro-Wilk test was used to demonstrate whether the investigated continuous variables were normally distributed. Therefore, continuous variables with and without normal distribution were presented as mean values \pm standard deviations or as medians and their interquartile ranges, respectively. Depending on the presence or absence of normal distribution, intergroup comparisons were performed with the Student's t-test for independent samples or the Mann-Whitney unpaired rank sum test, whereas the Student's t-test for paired samples or the Wilcoxon matched-paired rank sum test were applied for comparisons within the groups. Independent categorical variables were compared using the $\chi^{2}$ test with the Yates' correction if required. Dependent qualitative data were assessed with the McNemar's test. Correlations were tested with the Spearman rank correlation test. A value of two sided $\mathrm{p}<0.05$ was regarded as statistically significant; $0.05 \leq p<0.1$ was considered a trend towards statistical significance, while $\mathrm{p} \leq 0.1$ was marked as non-significant (NS). The statistical analysis and sample size calculation were carried out using the Statistica 10.0 package (StatSoft, Tulsa, OK, USA) and MedCalc 12.0 (MedCalc Software, Mariakerke, Belgium) statistical software.

\section{Results}

\section{Study population}

A total of 151 individuals were screened with respect to their eligibility for the study and 74 patients were eventually enrolled in the study (Fig. 1). All study participants received allocated antiplatelet regimen. However, 3 patients were excluded from the primary analysis. Finally, 71 patients ( 46 and 25 patients in the prasugrel and clopidogrel groups, respectively) were included in the primary analysis.

Baseline demographic and clinical characteristics of the study population are provided in Table 1. Similarly, both groups were comparable in terms of angiographic and procedural characteristics. The only discrepancy between both study arms was less common baseline TIMI 3 flow in the prasugrel group (13 [28.3\%] vs. 13 [52.0\%]; p < 0.05).

None of the study participants developed new onset myocardial infarction or was diagnosed with definite stent thrombosis at 30-day follow-up. Symptoms of new onset chronic heart failure occurred in $14(30.4 \%)$ patients treated with prasugrel and $8(32.0 \%)$ participants receiving clopidogrel $(\mathrm{p}=\mathrm{NS})$. One $(2.2 \%)$ patient from the prasugrel group reported an increased tendency for bruising. This patient continued therapy with prasugrel throughout the study period. No other bleeding events occurred in our study participants.

\section{Platelet reactivity}

The median time between administration of the $600 \mathrm{mg}$ loading dose of clopidogrel and baseline blood sampling did not differ between the prasugrel and clopidogrel groups (21.0 [14.0-23.5] vs. 20.7 [13.5-23.0] h; $\mathrm{p}=\mathrm{NS}$ ).

Platelet counts evaluated simultaneously with the baseline VASP and MEA measurements were comparable in the overall groups of patients assigned to the prasugrel and clopidogrel regimen (222 [193-258] vs. 243 [198-298] × $10^{3} \times \mu \mathrm{L}^{-1}$; $\mathrm{p}=\mathrm{NS}$ ), as well as in the subgroups treated without GP IIb/IIIa inhibitors (223 [200-255] vs. 232 
Table 1. Baseline characteristics of the study population.

\begin{tabular}{|c|c|c|c|}
\hline Variable & $\begin{array}{l}\text { Prasugrel group } \\
\qquad(n=46)\end{array}$ & $\begin{array}{l}\text { Clopidogrel group } \\
(\mathrm{n}=25)\end{array}$ & $\mathbf{P}$ \\
\hline Age [years] & $58.0(54.0-65.0)$ & $58.0(56.0-64.0)$ & NS \\
\hline Gender (male/female) & $10(21.7 \%) / 36(78.3 \%)$ & $10(40.0 \%) / 15(60.0 \%)$ & NS \\
\hline \multicolumn{4}{|l|}{ Clinical presentation: } \\
\hline STEMI & $34(73.9 \%)$ & $16(64.0 \%)$ & NS \\
\hline NSTEMI & $8(17.4 \%)$ & $7(28.0 \%)$ & \\
\hline Unstable angina & $4(8.7 \%)$ & $2(8.0 \%)$ & \\
\hline Anterior location of STEMI & $15(44.1 \%)$ & $9(56.2 \%)$ & NS \\
\hline LVEF at discharge [\%] & $45.0(40.0-50.0)$ & $45.0(40.0-50.0)$ & NS \\
\hline Prior myocardial infarction & $11(23.9 \%)$ & $4(16.0 \%)$ & NS \\
\hline Prior $\mathrm{PCl}$ & $8(17.4 \%)$ & $4(16.0 \%)$ & NS \\
\hline Prior CABG & $2(4.3 \%)$ & $2(8.0 \%)$ & NS \\
\hline \multicolumn{4}{|l|}{ Risk factors of coronary artery disease: } \\
\hline Body mass index $\left[\mathrm{kg} / \mathrm{m}^{2}\right]$ & $27.2(24.0-30.4)$ & $27.4(25.3-29.9)$ & NS \\
\hline Diabetes mellitus & $12(26.1 \%)$ & $4(16.0 \%)$ & NS \\
\hline Arterial hypertension & $31(67.4 \%)$ & $12(48.0 \%)$ & NS \\
\hline Current smokers & $25(54.4 \%)$ & $15(60.0 \%)$ & NS \\
\hline History of smoking & $5(10.9 \%)$ & $3(12.0 \%)$ & NS \\
\hline Positive family history & $8(17.4 \%)$ & $8(32.0 \%)$ & NS \\
\hline $\mathrm{GFR}\left[\mathrm{mL} / \mathrm{min} / 1.73 \mathrm{~m}^{2}\right]$ & $93.9 \pm 22.5$ & $90.6 \pm 17.6$ & NS \\
\hline Total cholesterol (mg/dL) & $217.8 \pm 46.2$ & $221.7 \pm 45.4$ & NS \\
\hline LDL cholesterol [mg/dL] & $150.6 \pm 42.0$ & $148.1 \pm 38.8$ & NS \\
\hline HDL cholesterol [mg/dL] & $43.5(37.0-48.5)$ & $47.0(37.0-61.0)$ & NS \\
\hline Triglycerides [mg/dL] & $118.5(77.0-160.5)$ & $99.0(75.0-145.0)$ & NS \\
\hline \multicolumn{4}{|l|}{ Pharmacotherapy during hospitalization: } \\
\hline Statin use & $46(100.0 \%)$ & $25(100.0 \%)$ & NS \\
\hline Beta-blocker use & $46(100.0 \%)$ & $25(100.0 \%)$ & NS \\
\hline ACEI use & $46(100.0 \%)$ & $24(96.0 \%)$ & NS \\
\hline Proton pump inhibitors use & $8(17.4 \%)$ & $4(16.0 \%)$ & NS \\
\hline Calcium-channel blocker use & $4(8.7 \%)$ & $0(0.0 \%)$ & NS \\
\hline Glycoprotein Ilb/IIla inhibitor administration & $16(34.8 \%)$ & $5(20.0 \%)$ & NS \\
\hline
\end{tabular}

ACEI - angiotensin-converting-enzyme inhibitor; GFR - glomerular filtration rate calculated according to the MDRD equation; CABG - coronary artery bypass grafting; LVEF - left ventricular ejection fraction; NSTEMI - non-ST-segment elevation myocardial infarction; PCI - percutaneous coronary intervention; STEMI - ST-segment elevation myocardial infarction

[196-281] $\left.\times 10^{3} \times \mu \mathrm{L}^{-1} ; \mathrm{p}=\mathrm{NS}\right)$. Additionally, we found no significant correlation between both, the baseline PRI and ADP-induced platelet aggregation values, and the platelet count.

The baseline PRI was 60.1 [39.9-71.2]\%; 46 $(64.8 \%)$ and $37(52.1 \%)$ patients after pre-treatment with a $600 \mathrm{mg}$ loading dose of clopidogrel presented with PRI values above the thresholds of $50 \%$ and $60 \%$ indicating HTPR. Personalized antiplatelet treatment utilized in our study enabled adequate platelet inhibition both at $24 \mathrm{~h}$ and at 30 days in the vast majority of our study participants (Figs. 2-5). The prasugrel regimen successfully overcame HTPR on clopidogrel in the acute phase of ACS and maintained its antiplatelet potency in 30-day follow-up while continuation of clopidogrel therapy in patients with an initially appropriate response to a $600 \mathrm{mg}$ loading dose of clopidogrel led to sufficient platelet inhibition in a predominant proportion of patients. In details, the median PRI value after loading with $30 \mathrm{mg}$ of prasugrel sharply declined by $83 \%$ at $24 \mathrm{~h}$ and slightly, but significantly, rose between $24 \mathrm{~h}$ and 30 days (Fig. 2). However, the median PRI value at 30 days in the prasugrel group was merely equal to $40 \%$ of its baseline value. In contrast, median PRI values in the clopidogrel group were similar at baseline and at $24 \mathrm{~h}$ and then modestly rose at 30 days. As shown in Figure 4, treatment with prasugrel was associated in our study with a significantly lower 


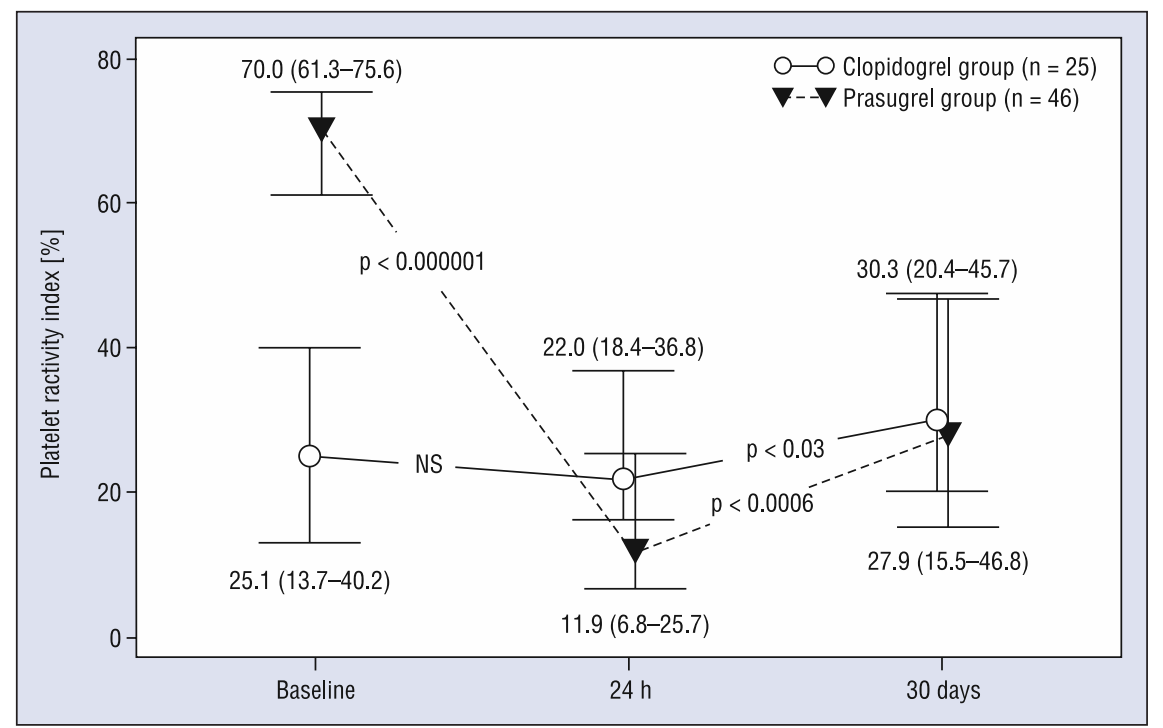

Figure 2. Platelet reactivity index according to the antiplatelet regimen. Data are presented as medians and interquartile ranges; $p$-values refer to differences within the same group between 2 consecutive sampling points.

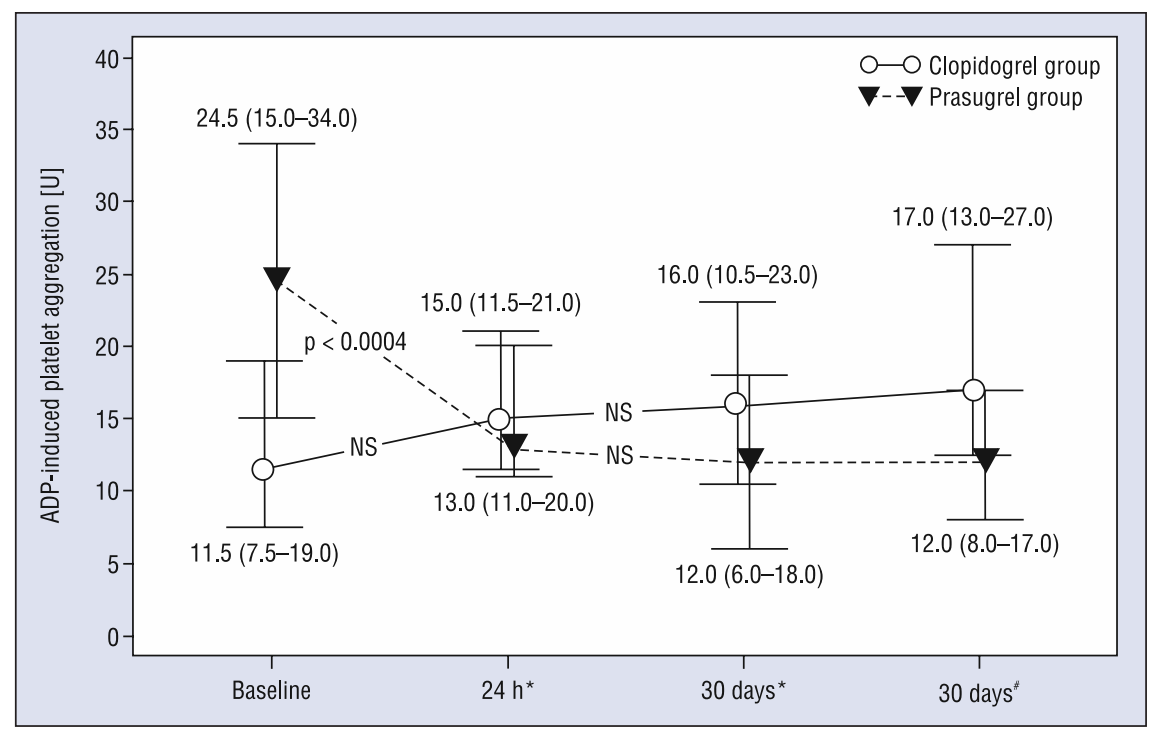

Figure 3. Adenosine diphosphate (ADP)-induced platelet aggregation according to the antiplatelet regimen. Data are presented as medians and interquartile ranges; $p$-values refer to differences within the same group between 2 consecutive sampling points; *indicates measurements performed exclusively in patients not treated with glycoprotein $\mathrm{Ilb} / \mathrm{Illa}$ inhibitors (20 and 30 patients in the clopidogrel and prasugrel groups, respectively); \#indicates measurements performed in all study participants (25 and 46 patients in the clopidogrel and prasugrel groups, respectively).

median PRI value at $24 \mathrm{~h}$ than therapy with clopidogrel. At baseline the numbers of patients with HTPR defined as the PRI value above the cut-offs of $50 \%$ and of $60 \%$ in the prasugrel and clopidogrel groups were: $46(100 \%)$ vs. $0(0 \%)$ for the cut-off of $50 \%$ and $36(78.3 \%)$ vs. $0(0 \%)$ for the cut-off of $60 \%$, at 24 h: $2(4.3 \%)$ vs. $2(8.0 \%)$ for the cut-off of $50 \%$ and $1(2.2 \%)$ vs. 1 (4.0\%) for the cut-off of
$60 \%$, and at 30 days: $8(17.4 \%)$ vs. $6(24.0 \%)$ for the cut-off of $50 \%$ and $2(4.3 \%)$ vs. $1(4.0 \%)$ for the cut-off of $60 \%$. Intra- and inter-group comparisons revealed a significant decrease in the proportion of patients treated with prasugrel and diagnosed with HTPR at $24 \mathrm{~h}$ when compared to their baseline status (a p-value for both cut-offs $<0.0001$ ). All other intra-group comparisons regarding the proportions 

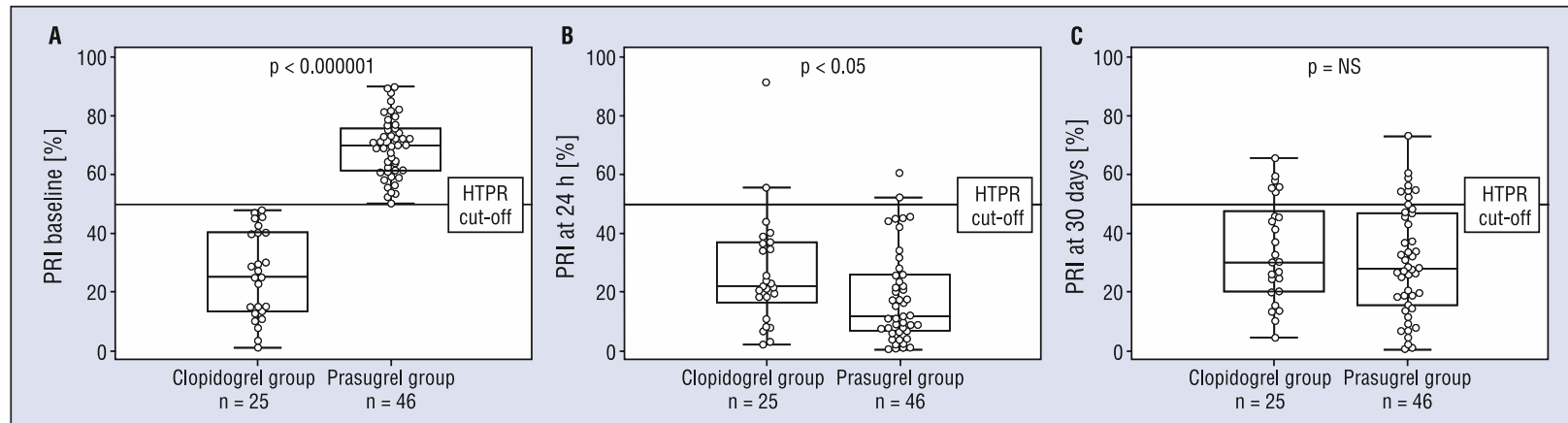

Figure 4. Individual patient values of platelet reactivity index at various sampling points according to the antiplatelet regimen. Boxes and whiskers represent medians, interquartile ranges and non-outlier ranges; $\mathbf{A}, \mathbf{B}$ and $\mathbf{C}$ refer to measurements performed at baseline, at $24 \mathrm{~h}$ and at 1 month, respectively; high on-treatment platelet reactivity (HTPR) cut-off in the figure indicates the threshold of $50 \%$; PRI — platelet reactivity index.

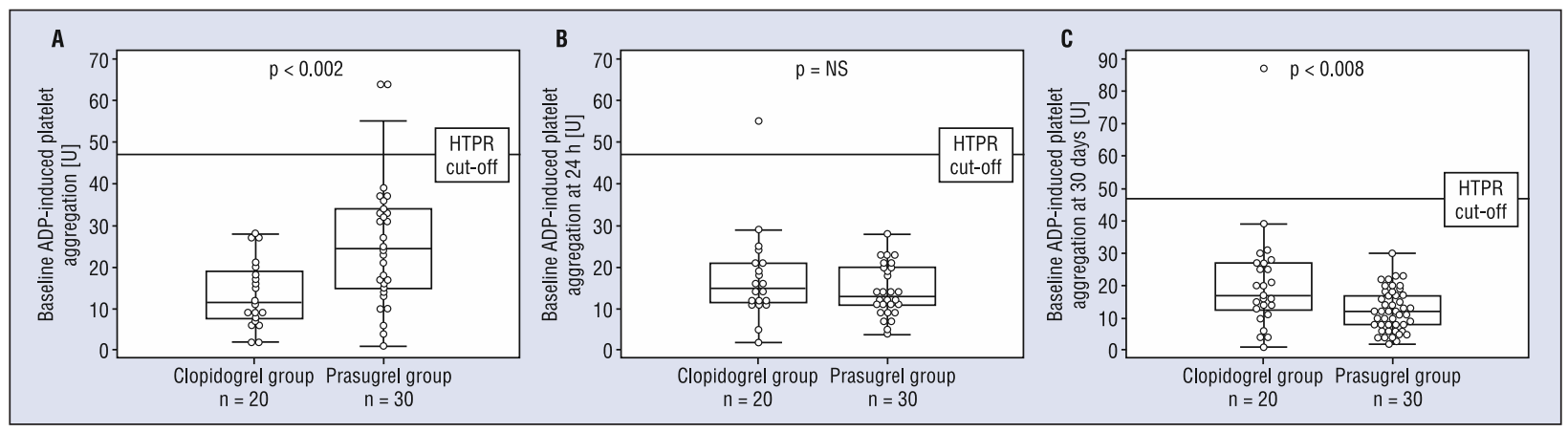

Figure 5. Individual patient values of adenosine diphosphate (ADP)-induced platelet aggregation at various sampling points according to the antiplatelet regimen. Boxes and whiskers represent medians, interquartile ranges and non-outlier ranges. A, B and C refer to measurements performed at baseline, at $24 \mathrm{~h}$ and at $1 \mathrm{month}$, respectively; high on-treatment platelet reactivity (HTPR) cut-off in the figure indicates the threshold of $46.8 \mathrm{U}$.

of patients with HTPR at 2 consecutive sampling points as well as inter-group comparisons of the proportions of patients with HTPR at $24 \mathrm{~h}$ and at 30 days indicated the lack of significant differences.

Baseline ADP-induced platelet aggregation assessed exclusively in patients not treated with GP IIb/IIIa inhibitors $(\mathrm{n}=50)$ was 17.5 [10.0-31.0] U. Three $(6.0 \%)$ of these patients after pre-treatment with a $600 \mathrm{mg}$ loading dose of clopidogrel presented with ADP-induced platelet aggregation values above both thresholds of $46.8 \mathrm{U}$ and $54 \mathrm{U}$, thus indicating HTPR. In general, the pattern of platelet inhibition evaluated with MEA followed tendencies observed with the VASP assay (Fig. 3), both in the prasugrel and clopidogrel groups. The median value of ADP-induced platelet aggregation after loading with $30 \mathrm{mg}$ of prasugrel decreased by $47 \%$ at $24 \mathrm{~h}$ and remained stable at 30 days. On the other hand, the measurements of ADP-induced platelet aggregation at all 3 sampling points were comparable in the clopidogrel group. As shown in Figure 5, tre- atment with prasugrel was associated in our study with a significantly lower median value of ADP-induced platelet aggregation at 30 days than therapy with clopidogrel. The baseline numbers of patients with HTPR defined as the ADP-induced platelet aggregation value above the cut-offs of $46.8 \mathrm{U}$ and of $54.0 \mathrm{U}$ in the prasugrel and clopidogrel groups were: $3(10.0 \%)$ vs. $0(0 \%)$ for the cut-off of $46.8 \mathrm{U}$ and $3(10.0 \%)$ vs. $0(0 \%)$ for the cut-off of $54.0 \mathrm{U}$, at $24 \mathrm{~h}: 0(0 \%)$ vs. $1(5.0 \%)$ for the cut-off of $46.8 \mathrm{U}$ and $0(0 \%)$ vs. $1(5.0 \%)$ for the cut-off of $54.0 \mathrm{U}$ and at 1 month: $0(0 \%)$ vs. $1(5.0 \%)$ for the cut-off of $46.8 \mathrm{U}$ and $0(0 \%)$ vs. $1(5.0 \%)$ for the cut-off of 54.0 U. Intra-group comparisons regarding the proportions of patients treated either with prasugrel or clopidogrel and presenting with HTPR defined according to any of the applied definitions at 2 consecutive sampling points as well as inter-group comparisons of the proportions of patients with HTPR at any of the sampling points showed no significant differences. 


\section{Discussion}

The main finding of our study is that prasugrel overcomes on-clopidogrel HTPR in the acute phase of interventionally treated ACS and maintains its antiplatelet potency in 30-day follow-up. Platelet inhibition on prasugrel therapy in our study participants with HTPR on clopidogrel was at least as effective as continuation of clopidogrel treatment in patients with an adequate response to a $600 \mathrm{mg}$ loading dose of clopidogrel. Additionally, our results indicate that the $600 \mathrm{mg}$ loading dose of clopidogrel fails to efficiently suppress platelet function in the acute phase of ACS according to the VASP assay in at least one half of patients undergoing PCI.

According to our best knowledge, this is the first study based on the VASP test in conjunction with another platelet function assay, reporting on the ability of prasugrel to overcome on-clopidogrel HTPR in ACS patients. In contrast to most of other trials [21-23], we also investigated the antiplatelet effects of both prasugrel loading and maintenance doses in ACS patients exhibiting HTPR on clopidogrel. Due to the unique property of the VASP assay to reflect selectively the inhibition of the $\mathrm{P}_{2} \mathrm{Y}_{12}$ receptor by antiplatelet agents, the VASP assay in contrast to other methods of platelet function monitoring may be applied in ACS patients treated with GP IIb/IIIa inhibitors. Previous studies on the efficacy of prasugrel in subjects with HTPR on clopidogrel therapy excluded persons receiving GP IIb/IIIa inhibitors and thus investigated highly selected populations [22, 24]. GP IIb/IIIa inhibitors are administered in individuals with unfavorable clinical characteristics including diabetic patients and those with extensive ST elevation myocardial infarction (STEMI), as well as in subjects with a high burden of intracoronary thrombus. These subsets of patients may potentially benefit most from intensified platelet inhibition. Similarly, in our study, patients on adjunctive therapy with GP IIb/IIIa inhibitors when compared with those receiving exclusively aspirin and clopidogrel were numerically more likely to present with HTPR at baseline according to the VASP assay. Not only does the VASP assay facilitate the risk stratification process in ACS patients undergoing PCI [10-14], but according to a recent study, it also provides the best reflection of the in vivo plasma level of the active metabolite of clopidogrel when compared with light transmission aggregometry, Verify-Now ${ }^{\circledR}$ system, whole blood aggregometry, and the IMPACT-R ADP test [25]. Numerous pharmacodynamic studies including unselected patients in terms of their baseline platelet reactivity demonstrated that pra- sugrel much stronger than clopidogrel, even with high doses of the latter (i.e. a loading dose of 600 $\mathrm{mg}$ and a maintenance dose of $150 \mathrm{mg}$ ), inhibits ADP-induced platelet aggregation [20, 26-28]. This intensified platelet inhibition by prasugrel is believed to account for the reduction of ischemic events when compared with clopidogrel therapy in ACS patients undergoing PCI which was shown in the TRITON-TIMI 38 trial [4]. However, recent studies indicated that the phenomenon of HTPR also refers to subjects treated with prasugrel [20, 21]. The prevalence of HTPR defined as the PRI value $\geq 50 \%$ measured $6-12 \mathrm{~h}$ after the administration of a $60 \mathrm{mg}$ loading dose of prasugrel among 301 ACS patients successfully treated with PCI in a multicenter French study was $25.2 \%$ [19]. This rate corresponds with the prevalence of HTPR on prasugrel at 30 days in the TRITON-TIMI 38 platelet substudy (24\% among 51 patients) [20], based on the same definition as applied by Bonello et al. [19].

In the first ever study demonstrating the efficacy of prasugrel in a broad range of post-PCI patients with HTPR on clopidogrel, Alexopoulos et al. [21] showed that prasugrel administered at a single dose of $10 \mathrm{mg}$ per day more effectively inhibited platelet aggregation than clopidogrel at a high dose of $150 \mathrm{mg}$ per day in 64 study participants. However, due to the specific study design (assessment of platelet reactivity at baseline, at 30 days and at 60 days) conclusions regarding the antiplatelet effect of the compared regimens must be restricted to the chronic therapy and cannot be applied to the acute setting when most of cases of stent thrombosis occur. The same research group demonstrated in another randomized study including 60 STEMI patients with HTPR diagnosed with the use of the Verify-Now ${ }^{\circledR}$ system $2 \mathrm{~h}$ after loading with $600 \mathrm{mg}$ of clopidogrel that prasugrel $(60 \mathrm{mg}$ loading dose/10 mg maintenance dose) provides faster and stronger platelet inhibition than a $150 \mathrm{mg}$ maintenance dose of clopidogrel. The authors excluded from the study patients treated with GP IIb/IIIa inhibitors and applied only 1 method of platelet function monitoring. Sardella et al. [23] demonstrated in the randomized, crossover RESET GENE trial, including 32 patients with HTPR on standard clopidogrel regimen who underwent elective coronary stenting, that treatment with a $10 \mathrm{mg}$ maintenance dose of prasugrel was associated with a significantly lower platelet reactivity than a $150 \mathrm{mg}$ maintenance dose of clopidogrel $(32.6 \pm 10.5$ vs. $47.9 \pm 20.9 \mathrm{U}$ according to MEA, $\mathrm{p}=0.028$ ). No patient treated with prasugrel exhibited HTPR 
defined as ADP-induced platelet aggregation with MEA > $45 \mathrm{U}$, whereas $9(28.1 \%)$ receiving high-dose clopidogrel still had HTPR $(\mathrm{p}=0.001)$.

Our results, when combined with the findings of other trials [21-24], provide a solid rationale for the conduction of a carefully designed randomized clinical trial aimed at testing potential clinical benefits of personalized prasugrel-based antiplatelet therapy. In our opinion, such a trial including ACS patients undergoing coronary stenting should compare a non-selective strategy (treatment of all patients with prasugrel) with a selective approach (switching to prasugrel therapy only in patients with HTPR on clopidogrel). We believe that personalized antiplatelet therapy in ACS patients may potentially lead to a reduction of bleeding complications and thus possibly translate into mortality benefits as well as save financial resources and in consequence increase the availability of new platelet $\mathrm{P}_{2} \mathrm{Y}_{12}$ inhibitors in low- and middle-income countries.

\section{Limitations of the study}

Several limitations to our study should be acknowledged. First, our study was performed in a non-randomized fashion. However, we believe that its design allows to prove our study hypothesis convincingly on the ability of prasugrel to overcome HTPR on clopidogrel in ACS patients and to clearly demonstrate that inhibition of platelet function with prasugrel in the setting of HTPR may be at least as effective as the antiplatelet effect of clopidogrel in non-HTPR patients. Furthermore, conduction of a reliable randomized study in the HTPR setting may create substantial difficulties. For example, in the TRIGGER-PCI trial, approximately one third of patients declined randomization when informed that HTPR had been detected [29]. Second, since we only analyzed surrogate end points, the hypothesis that prasugrel improves prognosis in interventionally treated ACS patients with HTPR should be verified in adequately powered clinical trials. Third, the limited sample sizes of our study preclude comprehensive sub-group analyses. Fourth, we did not analyze genetic polymorphisms involved in biotransformation of clopidogrel and prasugrel, nor did we assess active metabolites of thienopyridine derivatives [30]. Fifth, although the platelet VASP assay remains a very well validated method of platelet function monitoring, it requires an access to flow cytometry and a skilled technician to be performed.

\section{Conclusions}

Our study indicates that prasugrel overcomes HTPR on clopidogrel in the acute phase of interventionally treated ACS and maintains its antiplatelet potency in 30-day follow-up. Potential clinical benefits of personalized antiplatelet therapy based on prasugrel warrant further investigation in clinical trials in the ACS setting.

\section{Acknowledgements}

The authors would like to thank the residents and nurses of The Department of Cardiology and Internal Medicine, particularly Dr Malgorzata Molska and Mrs. Teresa Skibinska, for their valuable help in the enrolment process and blood samples collection. The present contribution is a project of Systematic Investigation and Research on Interventions and Outcomes (SIRIO)-MEDICINE, a group of senior scientists and fellows collaborating worldwide to pursue research and innovation in medicine.

Funding: The study was funded from departmental sources. Prasugrel manufacturer - Eli Lilly and Company - though generously providing the drug's free supply, throughout the whole study duration, had no influence nor input in the study design, data collection and interpretation, nor preparation of the manuscript.

Conflict of interest: Marek Kozinski - a speaker fee from AstraZeneca; Eliano Pio Navarese and Stefano De Servi — a speaker fee from Eli Lilly and Company; Jolanta Maria Siller-Matula — lecture or consultant fees from AstraZeneca, Daiichi Sankyo and Eli Lilly and Company. The rest of the authors declare no conflict of interest.

\section{References}

1. Tantry US, Bonello L, Aradi D et al. Consensus and future directions on the definition of high on-treatment platelet reactivity to adenosine diphosphate. J Am Coll Cardiol, 2013; 62: 2261-2273.

2. Siller-Matula JM, Delle-Karth G, Lang IM et al. Phenotyping vs. genotyping for prediction of clopidogrel efficacy and safety: The PEGASUS-PCI study. J Thromb Haemost, 2012; 10: 529-542.

3. Aradi D, Komócsi A, Vorobcsuk A et al. Prognostic significance of high on-clopidogrel platelet reactivity after percutaneous coronary intervention: Systematic review and meta-analysis. Am Heart J, 2010; 160: 543-551.

4. Wiviott SD, Braunwald E, McCabe $\mathrm{CH}$ et al. Prasugrel versus clopidogrel in patients with acute coronary syndromes. N Engl J Med, 2007; 357: 2001-2015. 
5. Navarese EP, Verdoia M, Schaffer A et al. Ischaemic and bleeding complications with new, compared to standard, ADP-antagonist regimens in acute coronary syndromes: A meta-analysis of randomized trials. QJM, 2011; 104: 561-569.

6. Kozinski M, Bielis L, Wisniewska-Szmyt J et al. Diurnal variation in platelet inhibition by clopidogrel. Platelets, 2011; 22: 579-587.

7. Wijns W, Kolh P, Danchin N et al. Guidelines on myocardial revascularization. Eur Heart J, 2010; 31: 2501-2555.

8. Aleil B, Ravanat C, Cazenave JP, Rochoux G, Heitz A, Gachet C. Flow cytometric analysis of intraplatelet VASP phosphorylation for the detection of clopidogrel resistance in patients with ischemic cardiovascular diseases. J Thromb Haemost, 2005; 3: 85-92.

9. Tóth O, Calatzis A, Penz S, Losonczy H, Siess W. Multiple electrode aggregometry: A new device to measure platelet aggregation in whole blood. Thromb Haemost, 2006; 96: 781-788.

10. Frere C, Cuisset T, Quilici J et al. ADP-induced platelet aggregation and platelet reactivity index VASP are good predictive markers for clinical outcomes in non-ST elevation acute coronary syndrome. Thromb Haemost, 2007; 98: 838-843.

11. Bonello L, Paganelli F, Arpin-Bornet M et al. Vasodilator-stimulated phosphoprotein phosphorylation analysis prior to percutaneous coronary intervention for exclusion of postprocedural major adverse cardiovascular events. J Thromb Haemost, 2007; 5: 1630-1636.

12. Blindt R, Stellbrink K, de Taeye A et al. The significance of vasodilator-stimulated phosphoprotein for risk stratification of stent thrombosis. Thromb Haemost, 2007; 98: 1329-1334.

13. Barragan P, Bouvier JL, Roquebert PO et al. Resistance to thienopyridines: clinical detection of coronary stent thrombosis by monitoring of vasodilator-stimulated phosphoprotein phosphorylation. Catheter Cardiovasc Interv, 2003; 59: 295-302.

14. Bonello L, Camoin-Jau L, Arques S et al. Adjusted clopidogrel loading doses according to vasodilator-stimulated phosphoprotein phosphorylation index decrease rate of major adverse cardiovascular events in patients with clopidogrel resistance: A multicenter randomized prospective study. J Am Coll Cardiol, 2008; 51: 1404-1411.

15. Sibbing D, Braun S, Morath $\mathrm{T}$ et al. Platelet reactivity after clopidogrel treatment assessed with point-of-care analysis and early drug-eluting stent thrombosis. J Am Coll Cardiol, 2009; 53: 849-856.

16. El Ghannudi S, Ohlmann P, Meyer N et al. Impact of P2Y12 inhibition by clopidogrel on cardiovascular mortality in unselected patients treated by percutaneous coronary angioplasty: A prospective registry. J Am Coll Cardiol Cardiovasc Interv, 2010; 3: 648-656.

17. Jeong YH, Bliden KP, Tantry US, Gurbel PA. High on-treatment platelet reactivity assessed by various platelet function tests: Is the consensus-defined cut-off of VASP-P platelet reactivity index too low? J Thromb Haemost, 2012; 10: 487-489.

18. Siller-Matula JM, Christ G, Lang IM, Delle-Karth G, Huber K, Jilma B. Multiple electrode aggregometry predicts stent thrombosis better than the vasodilator-stimulated phosphoprotein phosphorylation assay. J Thromb Haemost, 2010; 8: 351-359.
19. Bonello L, Pansieri M, Mancini J et al. High on-treatment platelet reactivity after prasugrel loading dose and cardiovascular events after percutaneous coronary intervention in acute coronary syndromes. J Am Coll Cardiol, 2011; 58: 467-473.

20. Michelson AD, Frelinger AL 3rd, Braunwald E et al. Pharmacodynamic assessment of platelet inhibition by prasugrel vs. clopidogrel in the TRITON-TIMI 38 trial. Eur Heart J, 2009; 30: 1753-1763.

21. Alexopoulos D, Dimitropoulos G, Davlouros P et al. Prasugrel overcomes high on-clopidogrel platelet reactivity post-stenting more effectively than high-dose (150-mg) clopidogrel: the importance of CYP2C19*2 genotyping. J Am Coll Cardiol Cardiovasc Interv, 2011; 4: 403-410.

22. Alexopoulos D, Galati A, Xanthopoulou I et al. Ticagrelor versus prasugrel in acute coronary syndrome patients with high on-clopidogrel platelet reactivity following percutaneous coronary intervention: a pharmacodynamic study. J Am Coll Cardiol, 2012; 60: 193-199.

23. Sardella G, Calcagno S, Mancone M et al. Pharmacodynamic effect of switching therapy in patients with high on-treatment platelet reactivity and genotype variation with high clopidogrel Dose versus prasugrel: the RESET GENE trial. Circ Cardiovasc Interv, 2012; 5: 698-704.

24. Alexopoulos D, Theodoropoulos KC, Stavrou EF et al. Prasugrel versus high dose clopidogrel to overcome early high on clopidogrel platelet reactivity in patients with ST elevation myocardial infarction. Cardiovasc Drugs Ther, 2012; 26: 393-400.

25. Bouman HJ, Parlak E, van Werkum JW et al. Which platelet function test is suitable to monitor clopidogrel responsiveness? A pharmacokinetic analysis on the active metabolite of clopidogrel. J Thromb Haemost, 2010; 8: 482-488.

26. Wiviott SD, Trenk D, Frelinger AL et al. Prasugrel compared with high loading- and maintenance-dose clopidogrel in patients with planned percutaneous coronary intervention: The Prasugrel in Comparison to Clopidogrel for Inhibition of Platelet Activation and Aggregation-Thrombolysis in Myocardial Infarction 44 trial. Circulation, 2007; 116: 2923-2932.

27. Angiolillo DJ, Badimon JJ, Saucedo JF et al. A pharmacodynamic comparison of prasugrel vs. high-dose clopidogrel in patients with type 2 diabetes mellitus and coronary artery disease: Results of the Optimizing anti-Platelet Therapy In diabetes MellitUS (OPTIMUS)-3 Trial. Eur Heart J, 2011; 32: 838-846.

28. Montalescot G, Sideris G, Cohen R et al. Prasugrel compared with high-dose clopidogrel in acute coronary syndrome. The randomised, double-blind ACAPULCO study. Thromb Haemost, 2010; 103: 213-223.

29. Trenk D, Stone GW, Gawaz M et al. A randomized trial of prasugrel versus clopidogrel in patients with high platelet reactivity on clopidogrel after elective percutaneous coronary intervention with implantation of drug-eluting stents: results of the TRIGGER-PCI (Testing Platelet Reactivity In Patients Undergoing Elective Stent Placement on Clopidogrel to Guide Alternative Therapy With Prasugrel) study. J Am Coll Cardiol, 2012; 59: 2159-2164.

30. Kubica A, Kozinski M, Grzesk G, Fabiszak T, Navarese EP, Goch A. Genetic determinants of platelet response to clopidogrel. J Thromb Thrombolysis, 2011; 32: 459-466. 